\title{
Correspondence
}

\section{Comment on: Patient physician communication}

\section{To the Editor}

The article by Alraddadi et al is timely and welcome. ${ }^{1}$ Effective patient physician communication is fundamental to successful clinical practice. ${ }^{2}$ Establishing rapport between physician and patient is paramount. This is achieved by attentive listening, showing empathy, understanding the patients' needs, allaying their anxiety, answering their worries and giving the patient hope. But at the same, the physician has to be honest and tells the truth. Reaching a diagnosis may not be difficult but getting the patient to accept treatment and/or changing their life style in stopping smoking, taking up regular exercise and changing diet require the physician to be convincing and gentle in making his advice. Treatment options, their risks and benefit need to be explained clearly in plain language to allow the patients to decide. This leads to a better shared decision making between the patient and physician. ${ }^{3}$

At the present time, most countries have become multi-ethnic and multi-cultural. The physician needs to understand and respect patients' culture.
Communication becomes difficult because of a language barrier. In such cases, advocates are needed to facilitate rapport. It would be interesting to see the findings if Alraddadi et $\mathrm{al}^{1}$ repeated the study with non-Saudi patients.

I believe patient physician communication skills should not only be taught in medical school and be part in the final clinical examination but also form part of postgraduate medical training. In many postgraduate examinations such as the MRCP (UK), communication skills are assessed vigorously.

\section{Ali S M Jawad Department of Rheumatology Royal London Hospital, Bancroft Road London, United Kingdom}

\section{References}

1. Alraddadi KS, Al-Adwani F, Taher ZA, Al-Mansour M, Khan M. Factors influencing patients' preferences for their treating physician. Saudi Med J 2020; 41: 866-873.

2. Travaline JM, Ruchinskas R, D’Alonzo Jr GE. Patient-physician communication: Why and How? J Am Osteopath Assoc 2005; 103: 13-18.

3. NICE. Shared decision making. [Update 2019 September 01; Accessed 2020 October 2]. Available from: https://www.nice. org.uk/advice/ktt23 